\title{
Direct Infusion Electrospray Ionization - Ion Mobility - High Resolution Mass Spectrometry (DIESI-IM-HRMS) for Rapid Characterization of Potential Bioprocess Streams
}

\author{
Sharon M. Munisamy, C. Kevin Chambliss, Christopher Becker \\ Department of Chemistry and Biochemistry, Baylor University, Waco, TX 76798, USA
}

\begin{abstract}
Direct infusion electrospray ionization-ion mobility-high resolution mass spectrometry (DIESI-IM-HRMS) has been utilized as a rapid technique for the characterization of total molecular composition in "whole-sample" biomass hydrolysates and extracts. IM-HRMS data reveal a broad molecular weight distribution of sample components (up to $1100 \mathrm{~m} / \mathrm{z}$ ) and provide trendline isolation of feedstock components from those introduced "in process." Chemical formulas were obtained from HRMS exact mass measurements (with typical mass error less than $5 \mathrm{ppm}$ ) and were consistent with structural carbohydrates and other lignocellulosic degradation products. Analyte assignments are supported via IM-MS collision-cross-section measurements and trendline analysis (e.g., all carbohydrate oligomers identified in a corn stover hydrolysate were found to fall within $6 \%$ of an average trendline). These data represent the first report of collision cross sections for several negatively charged carbohydrates and other acidic species occurring natively in biomass hydrolysates.
\end{abstract}

Key words: Ion mobility-mass spectrometry, Lignocellulomics, Biomass, Carbohydrates, Corn stover, Sorghum

\section{Introduction}

$\mathrm{U}$ nderstanding the correlation between sample composition and fuel production remains one of the greatest challenges facing the field of bio-based energy. Accordingly, detailed characterization of potential biofuel process streams is a critical step in optimizing biomass-to-fuel conversions, which are required for viable energy yields [1]. Most current biomass analyses target bulk components [2-5] or screen only a small fraction [6-13] of the total molecular composition. Moreover, these techniques can involve time-

Electronic supplementary material The online version of this article (doi:10.1007/s13361-012-0382-2) contains supplementary material, which is available to authorized users.

Correspondence to: Christopher Becker; e-mail: C_Becker@baylor.edu consuming preparation steps, extended analysis times, and multiple analyses for different compound classes [6-8, 1014]. Currently, there are relatively few examples of analyses applied to "whole-sample" biomass. Fluorescence and nearinfrared spectroscopic techniques coupled with multivariate statistics [2,3] and ${ }^{13} \mathrm{C}-\mathrm{NMR}$ spectroscopy [4] have been employed for rapid determination of whole-sample biomass hydrolysate. However, such techniques are best suited for providing scoping and/or macro-component (i.e., bulk) information on biomass samples. One approach targeting a whole-sample molecular-level understanding of biomass composition was recently reported by Helm and coworkers [5], in which flow injection electrospray - mass spectrometry (FIE-MS) was employed to identify several organic acids and carbohydrates in a dilute-acid pretreated biofeedstock. Although FIE-MS permits monitoring of multiple compound classes in a single analysis, collection of only 
nominal mass data in the absence of a separation technique can lead to uncertainty in analyte identification and loss of relevant signal in the presence of impurities (e.g., non-native sample components such as surfactants) that are inherent in sample generation.

Ion mobility - mass spectrometry (IM-MS) separates gas phase ions by size, shape, and $\mathrm{m} / \mathrm{z}$, offering an additional degree of separation over mass spectrometry alone. IM-MS has been applied as an effective tool for complex sample analysis in several fields, including proteomics [15-17], petroleomics [18-20], and metabolomics [21-23], and has the ability to separate ions based on differences in chemical class [24-27] and to distinguish between various molecular conformations and geometries [16, 18-20, 28]. IM-MS has also been utilized in small molecule analyses, including separation of pharmaceutical drug formulations [29, 30], chiral amino acid enantiomers [31], alkaloid stereoisomers [32], trace analysis of drugs [33], and screening for chemical warfare agents [34-36].

Despite the wide application of IM-MS to many complex matrices, its potential for use in analysis of samples derived from biomass feedstocks has not been investigated. A few analytes common to many biomass samples have been independently investigated with IM-MS (often using commercially available standards), including acetic acid [21] and several carbohydrates [21, 25-27, 37-39], such as 5- and 6carbon mono/oligosaccharides. However, in almost all of these studies, only positive ions have been analyzed, which is contrary to established mass spectrometry protocols for biomass analysis [5, 14]. Because of the diversity of structural components in biomass and ion mobility's potential for complex sample separation, IM-HRMS is an attractive candidate for whole-sample analysis of biomass hydrolysates and extracts.

Herein, we present direct infusion electrospray ionization - ion mobility - high resolution mass spectrometry (DIESI-IM-HRMS) as a novel approach to whole sample biomass analysis. DIESI-IM-HRMS is applied towards the identification and structural confirmation of multiple components in biomass samples. Our results indicate that this technique has the potential to separate and identify carbohydrates and other sample components from those introduced during pretreatment and sample preparation based on a combination of collision cross section (CCS) and HRMS analysis. We present, for the first time, collision cross sections for negatively charged carbohydrates and other analytes occurring natively in biomass samples. IM-MS correlations for structural carbohydrate oligomers in a bio-matrix have also been established to aid rapid identification of potential carbohydrates in a sample. While other techniques involve labor-intensive sample clean-up steps (e.g., multiple liquid and/or solid phase extractions [6]) or time-consuming chromatographic separation prior to MS analysis [6, 7, 13], DIESI-IM-HRMS is demonstrated to offer a high-throughput approach for rapid screening of whole-sample hydrolysates.

\section{Experimental}

\section{Chemicals and Reagents}

Xylobiose and xylotetraose were purchased from Megazyme International, Ireland (Wicklow, Ireland). All other chemicals were reagent grade or better and purchased from standard commercial vendors (i.e., Sigma-Aldrich, St. Louis, MO, USA or Fisher Scientific, Waltham, MA, USA). Distilled water was purified and deionized to $18.2 \mathrm{M} \Omega$ with a Barnstead Nanopure Diamond UV water purification system (Dubuque, IA). Dried sorghum feedstock was received from Dr. William L. Rooney, Department of Soil and Crop Sciences, Texas A\&M University (College Station, TX, USA). Corn stover hydrolysate (dilute-acid pretreated) was obtained from the National Renewable Energy Laboratory (NREL, Golden, CO, USA).

\section{Preparation of Standards and Generation of Aqueous Extract and Hydrolysate Samples}

Individual stock solutions of carbohydrates and organic acids were prepared at $100 \mathrm{ppm}$ by dissolving the neat chemical in water. From the stock solution, a $15 \mathrm{ppm}$ solution of each standard was prepared by serial dilution; these solutions were used for all analyses of standard compounds. A minimal volume of methanol was used in aiding dissolution of certain organic acids in stock solutions.

An aqueous extract of sorghum was prepared via accelerated solvent extraction (ASE-200; Dionex Corp., Sunnyvale, CA, USA) as previously reported [11]. Biomass hydrolysates were generated by pretreating sorghum with $0.7 \% \mathrm{H}_{2} \mathrm{SO}_{4}$ (wt/wt) using an ASE pretreatment protocol [40]. Briefly, operation conditions were as follows: $1 \mathrm{~g}$ of biomass per $11-\mathrm{mL}$ cell, temperature: $195{ }^{\circ} \mathrm{C}$, pressure: $1500 \mathrm{psi}$, preheat time: $9 \mathrm{~min}$, heat time: $9 \mathrm{~min}$, static time: $24 \mathrm{~min}$, purge time: $120 \mathrm{~s}$, flush volume: $150 \%$ of cell, and cycles: 1 . All samples were diluted 1:10 with purified water and filtered using a $25-\mathrm{mm}$ syringe filter with a $0.2 \mu \mathrm{m}$ nylon membrane (Pall Corp., Port Washington, NY, USA) before analysis.

\section{DIESI-IM-HRMS Analysis}

All analyses were performed with a Synapt HDMS ion mobility - mass spectrometer (Waters, Manchester, UK) equipped with an electrospray ionization source operated in negative mode. For all analyses except those involving collision-cross-section measurements, IMS separations were performed with a traveling-wave height of $8.0 \mathrm{~V}$ and applied voltages of 6.0 and $4.0 \mathrm{~V}$, respectively, to the trap and transfer cells. Collision-induced-dissociation (CID) experiments were conducted by increasing the voltage across the trap cell from $6.0 \mathrm{~V}$ to an optimized value between 20 and $30 \mathrm{~V}$ to produce abundant fragment ions across the $\mathrm{m} / \mathrm{z}$ range of interest. During IMS separations, a nitrogen bath gas was introduced into the IM cell at a flow rate of $30 \mathrm{~mL} / \mathrm{min}$ for most analyses providing a typical read-back pressure of 
0.68 mbar (when monitored by a Pirani gauge adjacent to the IMS cell). Where noted, a lower flow rate (i.e., $25 \mathrm{~mL} / \mathrm{min}$, 0.59 mbar) was used to compare ion mobility profiles of various analytes over a narrow mass range.

As noted in text, the Waters TOF was operated in either W mode $\left(\mathrm{R} \sim 15,000\left[\Delta m / m / z_{0.5}\right]\right)$ to improve accuracy of exact mass measurements or in $\mathrm{V}$ mode $(\mathrm{R} \sim 9000)$ to enhance instrumental sensitivity. Leucine enkephalin was used for instrument calibration and as a lock mass ion as described by Wolff et al. [41]. A $2 \mathrm{ng} / \mu \mathrm{L}$ lockspray solution was infused at 10 $\mu \mathrm{L} / \mathrm{min}$ and $1 \mathrm{~s}$ of lockspray data was collected for every $10 \mathrm{~s}$ of sample data. The $[\mathrm{M}-\mathrm{H}]^{-}$ion for leucine enkephalin $(\mathrm{m} / \mathrm{z}$ 554.2615 ) was used as a lock mass in post-run data processing. All theoretical masses were calculated using masses for ${ }^{12} \mathrm{C},{ }^{1} \mathrm{H}$, and ${ }^{16} \mathrm{O}$ isotopes listed in an International Union of Pure and Applied Chemistry (IUPAC) technical report [42]. All arrival time distributions were obtained by integrating the monoisotopic peak of a given analyte in the 2D IM-MS spectra and are presented uncorrected, as obtained from software (i.e., analyte arrival times are equal to the sum of their mobility drift time and time spent in transit between the drift cell and TOF detector).

\section{Collision-Cross-Section Measurements}

Collision cross sections of analytes were measured following the protocol reported by Ruotolo and coworkers [43]. [M- H] $]^{-}$ions generated from raffinose, melezitose, and $\alpha$-cyclodextrin standards, as well as fragment ions of $m / z 221$ and 323 resulting from collision-induced dissociation of $\alpha$-cyclodextrin and melezitose, respectively, were used as calibrants for collision-cross-section measurements. Collision cross sections for the calibrants were taken from literature and were 199.1, 196.4, 179.1, 134.1, and $163.2 \AA^{2}$ for raffinose, melezitose, $\alpha$-cyclodextrin, $m / z 221$, and $\mathrm{m} / \mathrm{z} 323$ fragment ions, respectively [39]. Accurate calibration was verified by re-measuring the collision cross sections of the standards using the generated calibration curve and assuring less than $1.0 \%$ deviation from reported values. IM-MS data were collected for standards and analytes at six different ion mobility wave heights (every $0.5 \mathrm{~V}$ in the range 5.5-8.0 V) at an ion mobility bath gas flow rate of $25 \mathrm{~mL} / \mathrm{min}$. Collision cross sections are reported as an average over all wave heights, with the exception of (1) ions having a molecular weight $<200 \mathrm{~m} / \mathrm{z}$ at larger wave heights, where field-strength-dependent effects were present and (2) ions having a molecular weight $>544 \mathrm{~m} / \mathrm{z}$ at smaller wave heights, where analyte distributions were outside of the ion mobility window. Arrival times used in collisioncross-section measurements were obtained from the monoisotopic peak of each analyte.

\section{Results and Discussion}

\section{Characterization of IM-MS Spectra of Biomass Pretreatment Hydrolysates and Aqueous Extracts}

Feasibility of DIESI-IM-HRMS for analysis of biomass samples was evaluated using two sample types derived from a sorghum feedstock: (1) a dilute-acid pretreatment hydrolysate and (2) an aqueous extract. These samples were selected as representative of the relatively complex matrices typically encountered in bioprocess workflows. Although pretreatment hydrolysates have long been a subject of investigation, relatively little is known about their total molecular composition. On the other hand, the relevance of aqueous extracts to valuations of potential energy-yield from a feedstock has only recently been realized [44].

Representative data for IM-MS analysis of a pretreatment hydrolysate and an aqueous extract is shown in Figure 1. Note that data in Figure 1 were collected over $2 \mathrm{~min}$. Compared with traditional chromatographic separations of biomass hydrolysates $[6,10,13,45]$, a 2 -min analysis time represents a 10 - to 20 -fold increase in throughput. In addition, these data exhibit an increase in peak capacity from $\sim 79,000$ with HRMS-only analysis to $\sim 350,000$ with IM-HRMS. The total ion signal observed via IM-MS analysis is separated into three distinct regions (indicated by trendline overlays I-III in Figure 1). Ions in trendlines I and II were singly-charged, whereas trendline III contained doubly-charged ions. In both hydrolysates and extracts, ion signal is observed across all arrival times and up
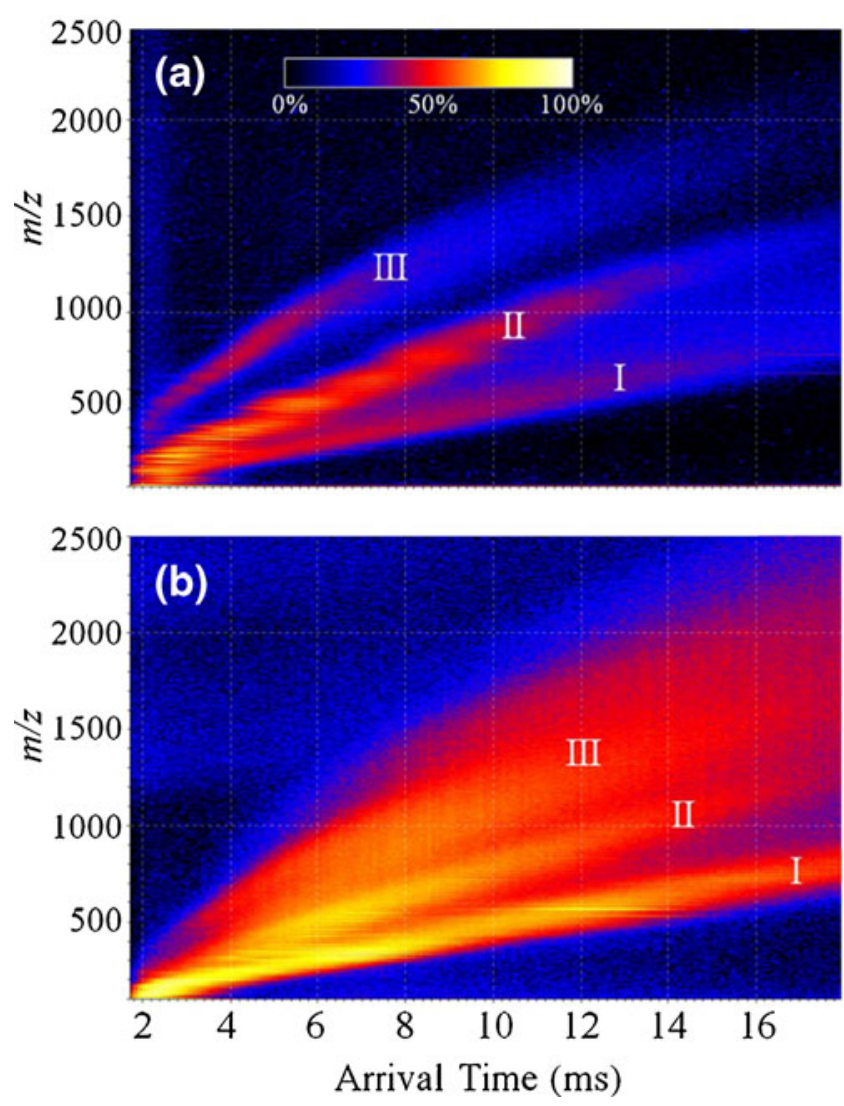

Figure 1. DIESI-IM-HRMS plot of $m / z$ versus arrival time for negative ions resulting from 1:10 dilutions of (a) $0.7 \%$ $\mathrm{H}_{2} \mathrm{SO}_{4}$-pretreated sorghum hydrolysate with three distinct trendlines (I, II, and III) and (b) aqueous extract of sorghum with two distinct trendlines (I and II) and a region with several unresolved trendlines (III) 
to $\sim 2000 \mathrm{~m} / z$. Note that the components typically monitored in biomass hydrolysates and extracts (i.e., organic-acid degradation products and small carbohydrates) are less than $250 \mathrm{~m} / z$. However, data in Figure 1 reveal that these analytes would only represent a small fraction of total sample composition, as a majority of ion signals fall outside of that mass range.

IMS separation enables MS signal to be isolated for specific trendlines. Data representative of the primary analyte signal in the two dominant trendlines (I and II, Figure 1) are shown in Figure 2. A number of carbohydrates and lignin degradation products were identified in trendline I by exact mass (Table S1, Supplemental Data) and collisioninduced dissociation (e.g., Figure S1 and Supporting Text). Despite the complexity of the sample and lack of prefractionation, experimental exact masses were found to be of reasonable quality matching the theoretical exact mass of assigned compounds with an average error of $3.0 \mathrm{ppm}$. It is important to note that all of these analytes were observed to occur along the bottom trendline (e.g., Figure 1a, I). Chemical formulas for other unidentified components in this

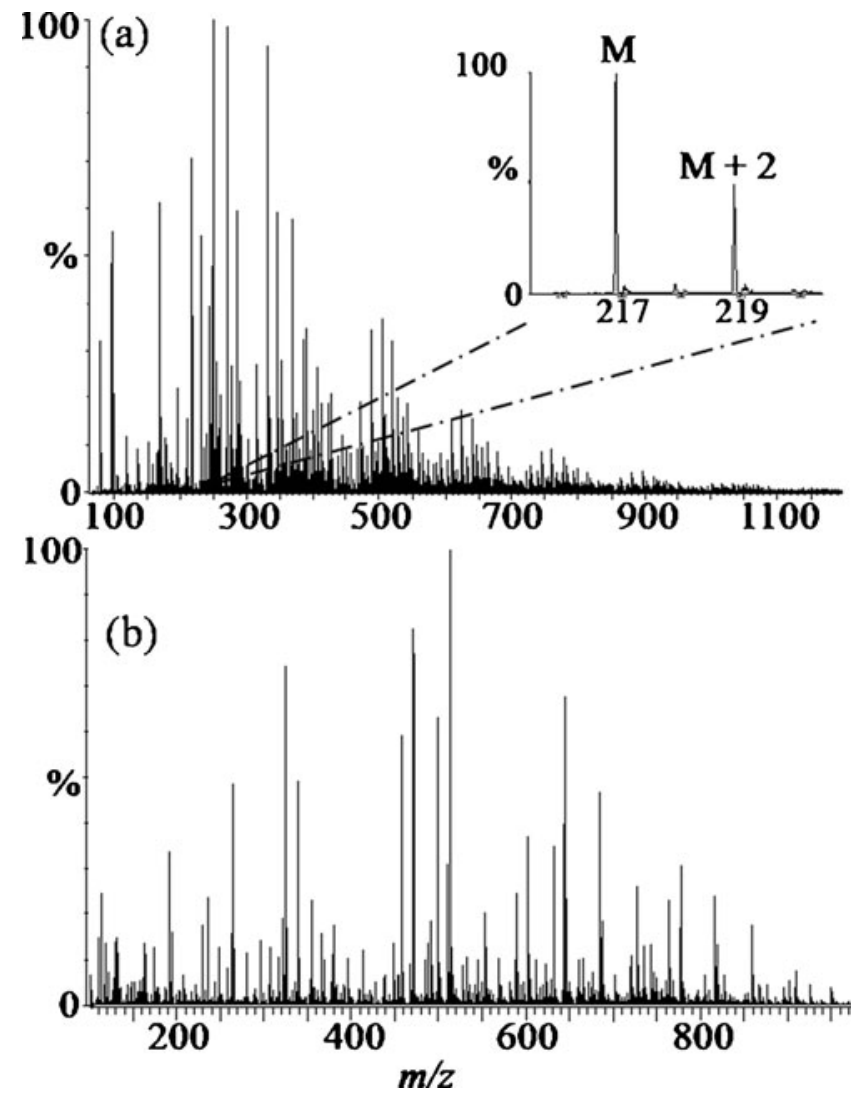

Figure 2. High-resolution mass spectra representative of the major trendlines observed for DIESI-IM-HRMS analysis of biomass pretreatment hydrolysates for (a) mobility-resolved salts corresponding to ion signal in trendline II and (b) organic species of lignocellulosic origin corresponding to ion signal in trendline I (see Figure 1). Inset shows isotopic spacing representative of most ions in trendline II trendline were also organic containing $\mathrm{C}, \mathrm{H}$, and $\mathrm{O}$. However, no organic species were observed in trendline II. The mass spectra of trendline II (Figure 1a) was extracted and is shown in Figure 2a. Most intense ions in these data exhibit a strong $\mathrm{M}+2$ isotope (Figure $2 \mathrm{a}$, inset), consistent with the presence of a $\mathrm{Cl}$ or $\mathrm{S}$ atom in these analytes (e.g., in the form of $\mathrm{Cl}^{-}$or $\mathrm{HSO}_{4}^{-}$adducts). Interpretation of $\mathrm{CID}$ data and chemical formulae determined from HRMS resulted in assignment of this trendline to singly-charged inorganic ion clusters (e.g., of the form $\left[\mathrm{K}_{2 \mathrm{n}-1}(\mathrm{X})_{\mathrm{n}}\right]^{-}$, $\left[\mathrm{K}_{\mathrm{n}}(\mathrm{X})_{\mathrm{n}+1}\right]^{-},\left[\mathrm{Na}_{2 \mathrm{n}-1}(\mathrm{X})_{\mathrm{n}}\right]^{-}$, etc., where $\mathrm{X}$ is typically a sulfate derivative or chloride). These inorganic ions are likely the result of the dilute-sulfuric acid pretreatment process, as they were also present in a control experiment, where dilute-acid ASE pretreatment was performed in an empty cell (i.e., in the absence of a feedstock). It is important to note that $\mathrm{HSO}_{4}{ }^{-}$adducts of carbohydrates and lignin degradation products were found to fall on the bottom trendline along with the non-adducted biomass analytes. However, no peaks were detected in the bottom trendline that were purely ionic/inorganic. Although ion signal in trendline III is too weak to achieve adequate accurate mass data, this signal's correlation between $\mathrm{m} / \mathrm{z}$ and mobility drift time is consistent with doubly charged salt adducts (in the case of Figure 1a, III) and both doubly charged salt adducts and doubly charged organic species in the broad upper regions of Figure 1b, III.

The persistence of inorganic salts and surfactants is especially problematic for MS analysis of routine biomass hydrolysates where pretreatment with dilute-sulfuric acid is among the most popular approaches [46]. In the absence of IMS separation, chemical noise almost fully obscured ion signal that was observed in trendline I (Figure 1a). However, data in Figures 1 and 2 demonstrate that the two-dimensional IM-MS separation enables signal resulting from inorganic salt adducts to be resolved from target organic species

\section{Evaluation of IM-HRMS Separation of Biomass Components}

The utility of IM-HRMS over low-resolution mass spectrometry or even HRMS only for whole-sample biomass analysis can be further evaluated through closer inspection of sample data. For example, a majority of the carbohydrates identified in these samples (Table S1, Supplemental Data) were observed to have another partially resolved ion occurring at the same nominal $\mathrm{m} / \mathrm{z}$ (separated by as little as $16 \mathrm{mDa}$ ). Figure 3 shows the IM-HRMS data for a dilute-acid pretreated corn stover hydrolysate in the range of a carbohydrate oligomer (xylotetraose, $545.1714 \mathrm{~m} / \mathrm{z}$ ) identified in the sample. These data (Figure 3, left projection) reveal two peaks with the same nominal mass but differing by $0.0711 \mathrm{~m} / \mathrm{z}$ (i.e., 545.1003 and 545.1714) and highlight the need for HRMS when employing direct injection ESI for complex sample analysis. More significantly, IMS separation enabled baseline resolution of 


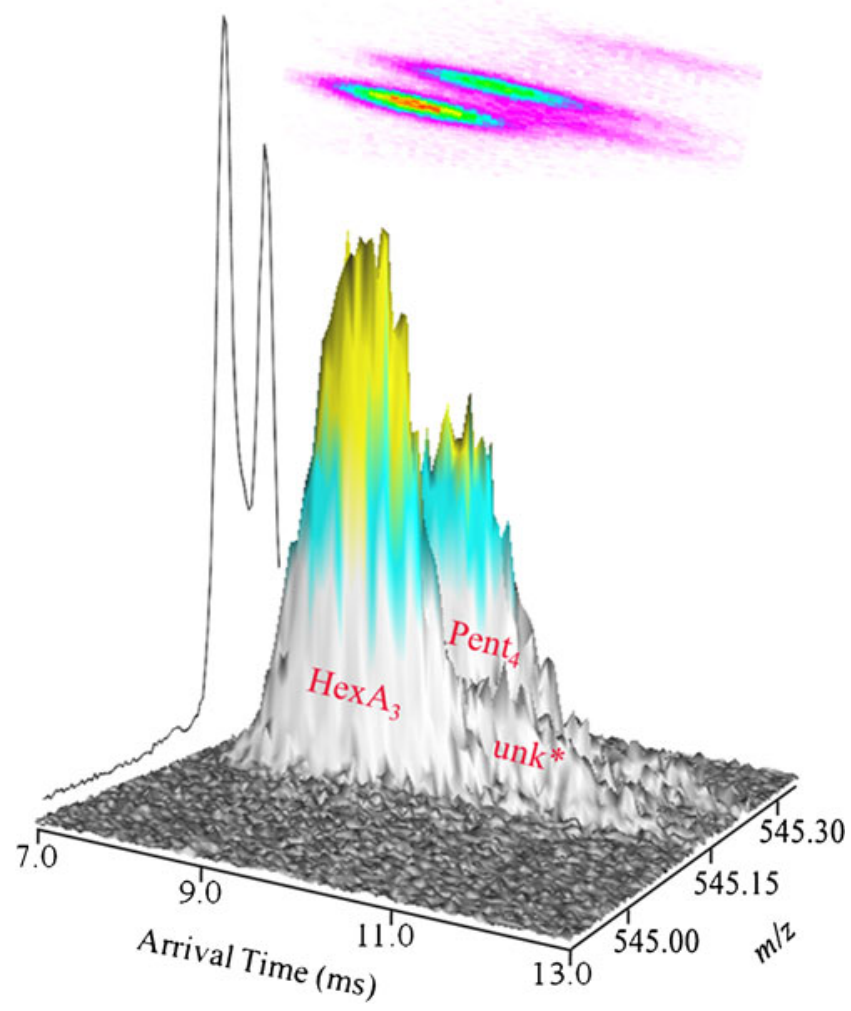

Figure 3. Three-dimensional plot of arrival time versus $\mathrm{m} / \mathrm{z}$ for a pentose tetramer $\left(\right.$ Pent $_{4}, 545.1714 \mathrm{~m} / \mathrm{z}$ ), a tentatively identified hexuronic acid trimer $\left(\mathrm{HexA}_{3}, 545.1003 \mathrm{~m} / \mathrm{z}\right)$, and an unknown component (unk* $545.14 \mathrm{~m} / \mathrm{z}$ ). These data demonstrate the potential for increasing component resolution when incorporating ion mobility as an alternate post-ionization separation. See text for details

the two main components (Figure 3, top projection) and also revealed the presence of a third species (i.e., "unk") that was not observable by HRMS analysis alone. The peak at $\mathrm{m} / \mathrm{z}$ 545.1003 is tentatively identified as a hexuronic acid trimer $\left(\mathrm{HexA}_{3}\right)$ based on exact mass (2.4 ppm error) and characteristic CID spectra. Arrival times of the two components identified in Figure 3 are centered at 8.874 and $10.005 \mathrm{~ms}$ for the 545.1003 and $545.1714 \mathrm{~m} / \mathrm{z}$ ions, respectively. The hexuronic acid oligomer with only three polymer units is observed at a shorter average arrival time than the 5 -carbon tetrasaccharide with four polymer units. In our experience using HRMS (resolving power up to $30 \mathrm{~K}$ ) for analysis of complex samples, we have frequently observed a negative impact on mass accuracy due to peak asymmetry resulting from unresolved analytes. These data in Figure 3 show IMS could facilitate accurate mass measurements by improving mass spectral peak purity. However, software that can calculate accurate mass based on three-dimensional data needs to be developed and/or standardized before such a benefit can be fully realized.

In addition to enhancing resolving power, DIESI-IM-HRMS can also be employed towards rapid fingerprinting of potential biofuel process streams. Chemical fingerprinting of lignocellulomic samples by MS alone can be particularly difficult owing to the high degree of structural diversity, which results in (potentially multiple) ion signal(s) at every nominal mass (e.g., Figure 4, top). However, data in Figure 4 (bottom) show unique arrival times at nominal $\mathrm{m} / \mathrm{z}$ in each sample, allowing rapid differentiation between samples and/or sample components. Such fingerprinting of biofuel process streams may be particularly useful when monitoring stream "health," assessing sample viability, and during chemometric feedstock valuations [14].

Both peak width and arrival times may be used to evaluate the presence of specific species. For example, exact mass identified a 5-carbon trisaccharide at $\mathrm{m} / \mathrm{z} 503$ in both corn stover and sorghum hydrolysates. Four common glucose trisaccharide isomers with the same chemical formula as the unknowns but varying in linkage position were analyzed with DIESI-IM-HRMS and compared with the broad IMS distributions in the real samples (Figure 5). Each ion was observed to have a unique arrival time distribution with a base peak width of about $2.2 \mathrm{~ms}$. Arrival times of the same $\mathrm{m} / \mathrm{z}$ in corn stover and sorghum fall between that of maltotriose and the other three isomers and are substantially broader (3.5-4.0 ms at the base) than the width of a single-component peak, indicating that multiple carbohydrate isomers may be present in the biomass samples (these data are described in more detail below). Note that improved IMS resolving power would be expected to substantially improve differentiation of isomers, improving the utility of the DIESI-IM-MS approach. For example, with an IMS resolution of $45(\mathrm{R}=\Omega / \Delta \Omega)$, which has been reported for currently available commercial instrumentation [47], melezitose and raffinose would be partially resolved

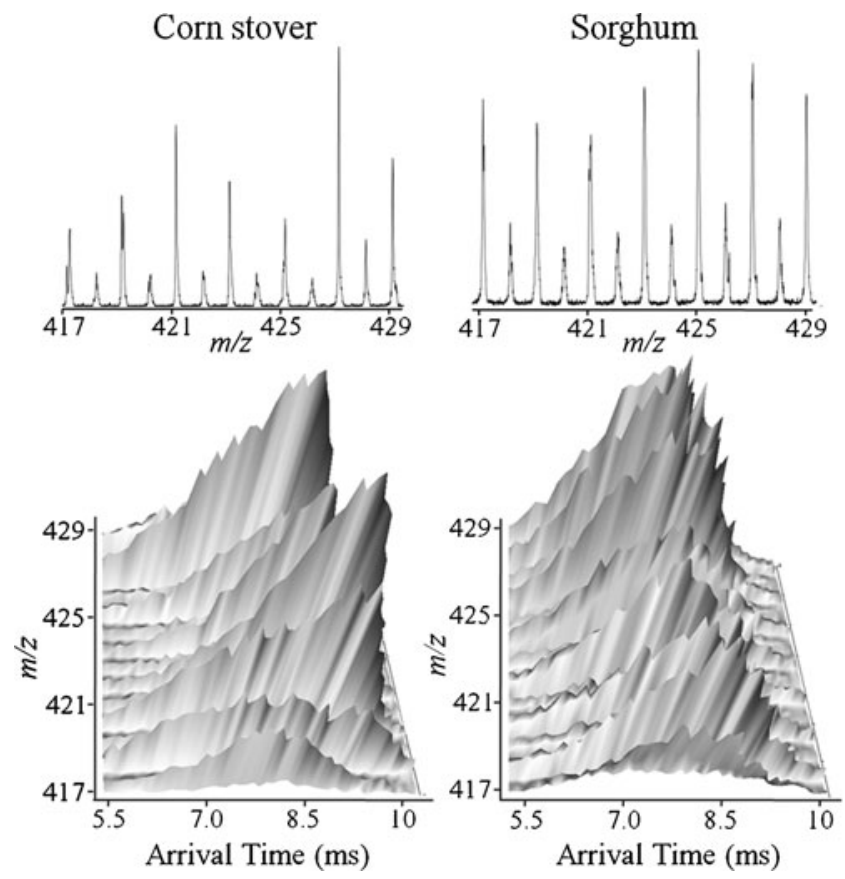

Figure 4. Representative HRMS (top) and IM-HRMS (bottom) data for corn stover (left) and sorghum (right) hydrolysates. Note the differences in ion arrival times for ions in the range $417-429 \mathrm{~m} / \mathrm{z}$, which is indicative of the different sample components in this range 


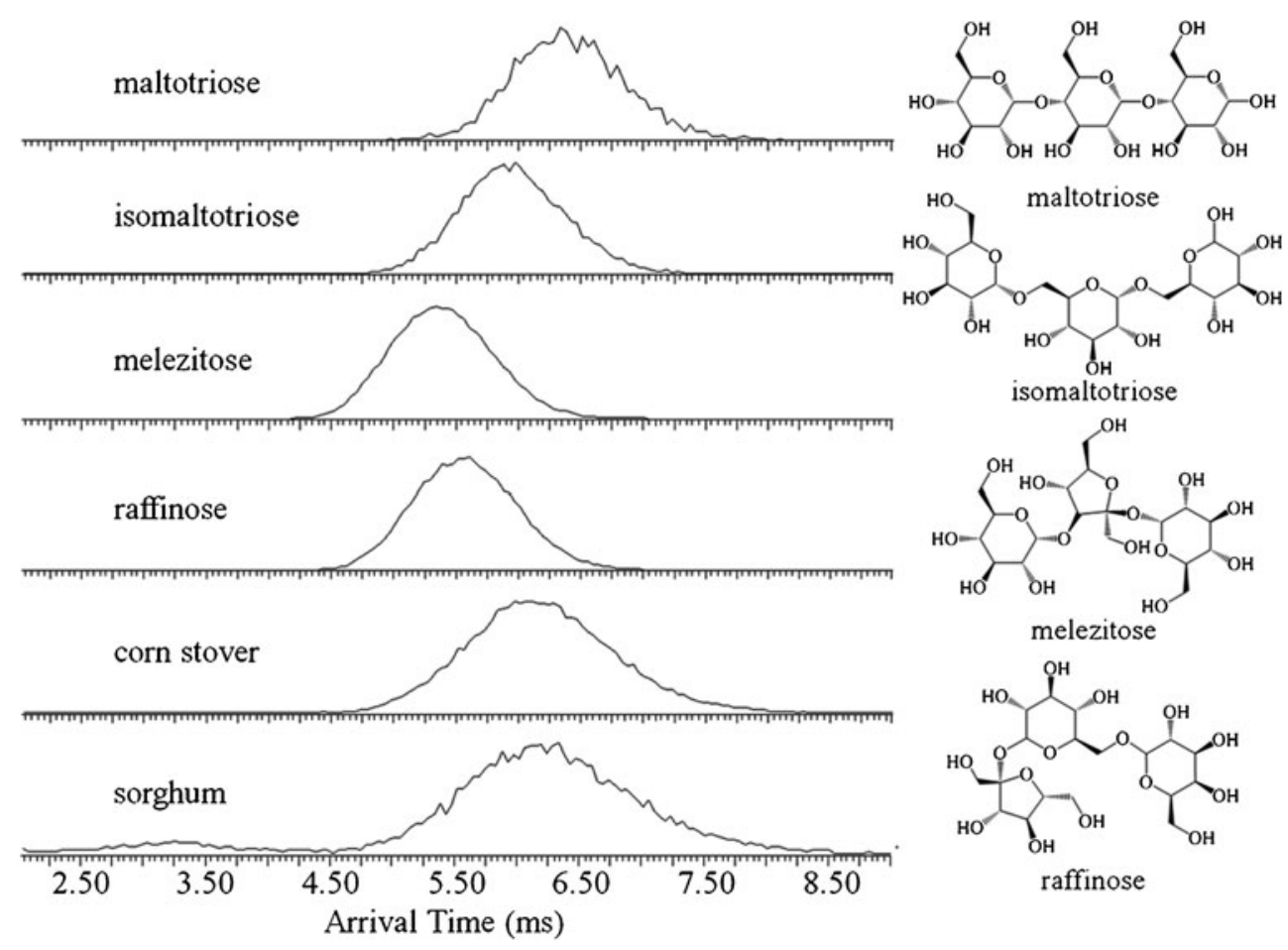

Figure 5. Arrival time distributions for the $[\mathrm{M}-\mathrm{H}]^{-}$ion of four 6-carbon trisaccharide isomer standards and the corresponding trisaccharide signal observed in corn stover and sorghum hydrolysates. Structures for the carbohydrate standards reported in this figure are shown on the right. Note that an IM gas flow rate of $25 \mathrm{~mL} / \mathrm{min}$ was used in the collection of these data

(overlapping at $\sim 1 / 3$ height with an approximate peak-to-peak resolution of 1) and the other isomers would be baseline resolved.

\section{Mass-Mobility Correlations of Carbohydrates in Biomass}

Utilizing mass-mobility correlations can be important for analyte determinations in complex samples. For example, when investigating a corn stover hydrolysate on a nominalmass mass spectrometer, Helm and researchers [5] observed a peak at $\mathrm{m} / \mathrm{z} 246.8$ that was identified as a pentose- $\mathrm{HSO}_{4}{ }^{-}$ adduct. In the mass spectrum of biomass hydrolysate used in this work, an intense peak at $\mathrm{m} / \mathrm{z} 246.85$ was also observed with a corresponding average arrival time of $2.62 \mathrm{~ms}$. Based on previous work [5], the analyst would be tempted to assume this analyte is the same pentose- $\mathrm{HSO}_{4}{ }^{-}$ion as reported previously. However, the mass and arrival time of this ion was observed to correspond to an analyte positioned on the middle trendline (i.e., Figure 1a, II), indicating that it is an inorganic ion. A less intense peak at $\mathrm{m} / \mathrm{z} 247.01$ and $4.24 \mathrm{~ms}$ was identified as a potential candidate for the pentose- $\mathrm{HSO}_{4}{ }^{-}$adduct, which was confirmed by exact mass. With unique mass-mobility correlations for different compound classes (e.g., carbohydrates and inorganic ions), identification of peaks with close or even the same molecular weight can be made with improved confidence without requiring additional analyses (e.g., LC-MS, CID, etc.). In the current work, a linear mass/mobility correlation was observed for all carbohydrate oligomers independent of ion type, for example, $[\mathrm{M}-\mathrm{H}]^{-},[\mathrm{M}+\mathrm{Cl}]^{-}$, and $\left[\mathrm{M}+\mathrm{HSO}_{4}\right]^{-}$ (Figure 6). All of the identified carbohydrate species were observed to fall within $6 \%$ of the average trendline, suggesting that any ion signal exhibiting greater deviation should be

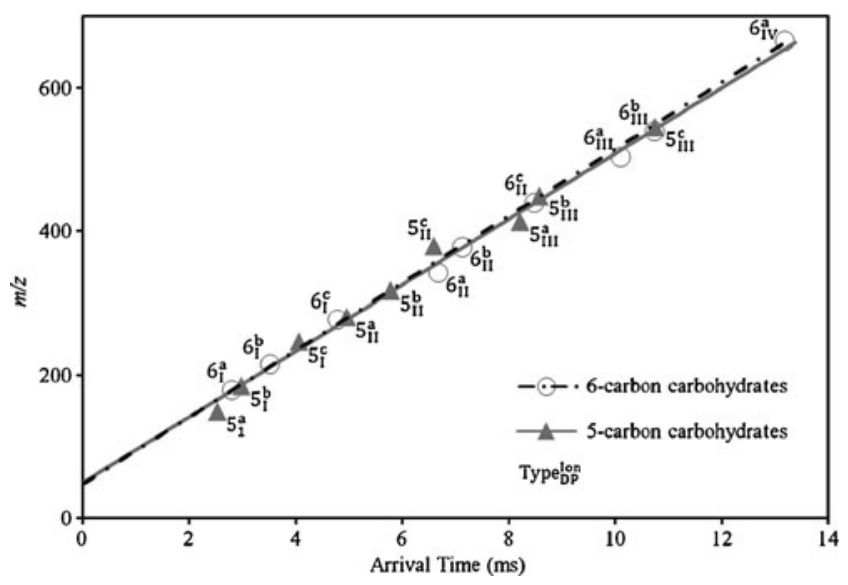

Figure 6. Mass-mobility correlations for 5- and 6-carbon carbohydrate ions observed in corn stover hydrolysate. The numbers "5" and "6" are added to the plot for easy identification of 5- and 6-carbon data points with superscripts and subscripts representing, respectively, the ion type and degree of polymerization. Superscript letters designate ion type as follows: (a) $[\mathrm{M}-\mathrm{H}]^{-}$, (b) $[\mathrm{M}+\mathrm{Cl}]^{-}$, and (c) $\left[\mathrm{M}+\mathrm{HSO}_{4}\right]^{-}$. Degree of polymerization (i.e., DP units) for each ion is indicated by the subscripts I, II, III, and IV, respectively, for the monomer through increasing polymer units 
carefully evaluated before making a positive carbohydrate assignment.

\section{Collision-Cross-Section Measurements of Biomass Analytes}

Comparison of ion mobility arrival times for standard and sample peaks can be useful in analyte identification. However, arrival times can vary between instruments and are dependent on various instrumental parameters. Collision cross sections provide an effective measure of an ion's gasphase size, which is constant for a given analyte assuming "low field" conditions are maintained. Thus, measurement of CCS can provide increased confidence in analyte assignments, particularly in the case of isomers. To generate representative data, collision cross sections of carbohydrate standards and select carbohydrate ions in a corn stover hydrolysate were measured. Table 1 shows CCS values for the $[\mathrm{M}-\mathrm{H}]^{-}$ions of xylose, glucose, and seven oligosaccharide standards. Generally, CCS values ranged from 110.4 to $258.2 \AA^{2}$ and were similar to those reported for the corresponding positive ions (i.e., $[\mathrm{M}+\mathrm{Na}]^{+}$) $[25,26,37,38]$. Negative-ion CCSs were $\sim 2 \%$ larger than literature values for xylose and glucose and between $7 \%-16 \%$ different for the other oligosaccharides. It is not known whether these differences in cross section result from differences in ion structure or

Table 1. Measured Average Collision Cross Sections for Negatively Charged Ions of Select Carbohydrate Standards (top) and Identified Carbohydrates in Corn Stover Hydrolysate (bottom). DP Represents the Number of Monomer Units in the Carbohydrate Polymer

\begin{tabular}{|c|c|c|c|c|c|}
\hline Analyte & DP unit & $m / z^{\mathrm{a}}$ & \multicolumn{3}{|c|}{ Collision cross section ${ }^{\mathrm{b}}\left(\AA^{2}\right)$} \\
\hline Standards & & & \multicolumn{3}{|l|}{$[\mathrm{M}-\mathrm{H}]^{-}$} \\
\hline Xylose & 1 & 149 & \multicolumn{3}{|l|}{110.4} \\
\hline Xylobiose & 2 & 281 & \multicolumn{3}{|l|}{150.3} \\
\hline Xylotetraose & 4 & 545 & \multicolumn{3}{|l|}{214.7} \\
\hline Glucose & 1 & 179 & \multicolumn{3}{|l|}{118.3} \\
\hline Maltose & 2 & 341 & \multicolumn{3}{|l|}{166.0} \\
\hline Maltotriose & 3 & 503 & \multicolumn{3}{|l|}{210.6} \\
\hline Isomaltotriose & 3 & 503 & \multicolumn{3}{|l|}{205.3} \\
\hline Melezitose & 3 & 503 & \multicolumn{3}{|l|}{197.1} \\
\hline Raffinose & 3 & 503 & \multicolumn{3}{|l|}{200.2} \\
\hline Maltotetraose & 4 & 665 & \multicolumn{3}{|l|}{227.2} \\
\hline Stachyose & 4 & 665 & \multicolumn{3}{|l|}{227.7} \\
\hline Maltopentaose & 5 & 827 & \multicolumn{3}{|l|}{258.2} \\
\hline \multicolumn{6}{|l|}{ Corn stover } \\
\hline \multirow[t]{4}{*}{ 5-Carbon } & 1 & 149 & 113.8 & 127.1 & 145.5 \\
\hline & 2 & 281 & 150.8 & 158.3 & 169.5 \\
\hline & 3 & 413 & 191.2 & 194.3 & 202.3 \\
\hline & 4 & 545 & 212.3 & 219.8 & 220.6 \\
\hline \multirow[t]{5}{*}{ 6-Carbon } & 1 & 179 & 120.6 & 136.6 & 144.7 \\
\hline & 2 & 341 & 167.9 & 185.4 & 185.4 \\
\hline & 3 & 503 & 210.1 & 215.2 & 216.9 \\
\hline & 4 & 665 & 235.7 & - & - \\
\hline & 5 & 827 & 256.6 & - & - \\
\hline
\end{tabular}

${ }^{\text {a }} m / z$ represents nominal mass for $[\mathrm{M}-\mathrm{H}]^{-}$ions. $m / z$ of chloride and bisulfate adducts in corn stover (not shown) are equivalent to the $m / z$ of $[\mathrm{M}-\mathrm{H}]^{-}+36$ and $+98 \mathrm{Da}$, respectively

${ }^{\mathrm{b}}$ Relative standard deviations for all CCSs are less than $1 \%$ from differences between the two approaches for measurement. Although it would not be unexpected for negative ions to adopt a substantially different structure than positive ions, the collision cross sections found in this work for negative trisaccharide ions exhibit the same trend (maltotriose> isomaltotriose $>$ raffinose $>$ melezitose) as CCSs for positive ions $[26,37,38]$.

A direct correlation between field strength in the traveling wave drift cell and a static DC field cannot be made to directly evaluate the low field limit during CCS measurements. In our work, CCSs were constant for all wave heights used to calculate the value. However, continued increase of wave heights beyond the values reported in this study did result in mass-dependent variation in observed CCS. Considering ion mobilities differ in high field versus low field conditions, we interpret the wave heights providing constant CCS values in our experiments to be indicative that the effective "field strength" was analogous to low field conditions in a classical DC drift cell experiment. In addition, it is reasonable that the low field limit for a given ion may be estimated by monitoring CCS with respect to wave height, and the possibility of using these data to accurately estimate effective ion temperatures is the subject of ongoing work in our laboratory.

CCS data for carbohydrates identified in the corn stover hydrolysate were determined for $[\mathrm{M}-\mathrm{H}]^{-},[\mathrm{M}+\mathrm{Cl}]^{-}$, and $[\mathrm{M}+$ $\left.\mathrm{HSO}_{4}\right]^{-}$ions and are reported in Table $1\left(\mathrm{Cl}^{-}\right.$and $\mathrm{HSO}_{4}{ }^{-}$ adducts of the 6-carbon tetra- and pentasaccharides were not observed in the hydrolysate). These CCS values have a similar range as the analyzed standards (113.8-256.6 $\left.\AA^{2}\right)$ and agree with the values for the $[\mathrm{M}-\mathrm{H}]^{-}$ions of the standard carbohydrates ( $\leq 3 \%$ difference), suggesting that the molecular structures of the standard analytes are probably the same as or similar to those analytes observed in corn stover. We note that direct comparison of $\mathrm{Cl}^{-}$and $\mathrm{HSO}_{4}{ }^{-}$adducts between the hydrolysate and the standards is not possible as they were not observed in the standard solutions. One exception to the agreement between the standards and analytes observed in corn stover is the CCSs of the 6-carbon trisaccharides. Four different 6-carbon trisaccharide isomer standards (see Figure 5 for structures and ion arrival times) were investigated in this work, and their collision cross sections range from 197.1 (melezitose) to 210.6 (maltotriose) $\AA^{2}$ (Table 1). It is important to note that the broad arrival time distribution for the 6-carbon trisaccharide in corn stover suggests the presence of multiple isomers. However, the average collision cross section of this peak $\left(210.1 \AA^{2}\right)$ is most similar to that of maltotriose $(<1 \%$ difference), indicating that maltotriose might be the most abundant isomer in the sample.

A particularly interesting feature of the collision-crosssection data for carbohydrates (Table 1) is that a significant difference in collision cross sections for the deprotonated and adducted $\left(\mathrm{Cl}^{-}\right.$and $\left.\mathrm{HSO}_{4}{ }^{-}\right)$ions is observed. In the absence of conformational change, ion adduction generally does not significantly alter the collision cross section of ions in large molecules (e.g., peptides and proteins), especially in the case 
of sodium or potassium adducts [48]. However, adducting ions have been observed to change CCSs for smaller molecules $<200 \mathrm{~m} / \mathrm{z}$ [up to $14 \%$ and $24 \%$ change in CCS with $\mathrm{Na}^{+}$and $\mathrm{Ca}\left(\mathrm{CH}_{3} \mathrm{COO}\right)^{+}$, respectively] [25, 34, 36]. It is currently not clear whether the observed change in cross section is due to conformational change or to the relative size of the anion. We note that the difference is larger for lower molecular weight carbohydrates (up to $32 \%$ ) than for higher molecular weight carbohydrates (as low as $2.4 \%$ ), which may indicate that the relative size of the adducting ion to the analyte is alone responsible for influencing the observed CCS. In addition, bisulfate adducts were found to have larger collision cross sections than chloride adducts, with the exception of the 6-carbon disaccharide and trisaccharide where the values are nearly equivalent.

Collision cross sections were also measured for select small molecule $(<200 \mathrm{~m} / \mathrm{z})$ biomass degradation products (i.e., aromatic acids and aldehydes). Collision cross sections of standards and corresponding ions found in corn stover and sorghum hydrolysates are listed in Table S1 (Supplementary Data), and illustrate the value of utilizing CCS measurements to aid identification of isomeric species with small structural differences. A comparison of cross sections between the standard and hydrolysate ions can provide additional information about sample composition. For example, homovanillic acid and syringaldehyde, which have cross sections of 130.6 and $126.7 \AA^{2}$, respectively, are both analytes that have been regularly observed in dilute-acid pretreatment hydrolysates during our analyses by LC-MS/MS. The cross sections of the ion having the same predicted chemical formula in corn stover and sorghum (127.3 and $127.2 \AA^{2}$, respectively) closely matches the cross section of syringaldehyde, indicating that the $\mathrm{m} / \mathrm{z}$ peak in the hydrolysates might belong to syringaldehyde. These data are in good agreement with quantitative determination of syringaldehyde and homovanillic acid in corn stover (by an LC-MS/MS method [9]), which indicates syringaldehyde is the most abundant isomer at $19.8 \mathrm{ppm}$ while homovanillic acid is only present at a much lower concentration (1.08 ppm).

For some isomers, CCS data can be less conclusive. The collision cross section for $\mathrm{C}_{7} \mathrm{H}_{6} \mathrm{O}_{4}$ in corn stover $\left(113.3 \AA^{2}\right)$ was between the cross sections for our standard $\mathrm{C}_{7} \mathrm{H}_{6} \mathrm{O}_{4}$ isomers $\left(111.1 \AA^{2}\right.$ and $115.6 \AA^{2}$, respectively, for 2,5- and 3,4-dihydroxybenzoic acids). The presence of both 2,5- and 3,4-dihydroxybenzoic acid were confirmed as present in the sample by LC-MS/MS analyses, suggesting the intermediate collision cross section of the unknown isomer in corn stover results from the presence of both of these ions. However, without complimentary analysis such as LC-MS or assessment of peak width (as described above), it could have been concluded that a different $\mathrm{C}_{7} \mathrm{H}_{6} \mathrm{O}_{4}$ isomer was in the sample. These data indicate that although small differences in isomer CCSs can be easily observed and reliably quantified in pure standards, IMS resolution is very important when more than one isomer may be present in a sample at similar intensity.
Evaluation of CCS for analyte determination does bring about a few interesting perspectives and/or concerns regarding the use of DIESI-IM-HRMS analysis for these sample types. First, note that while syringaldehyde and homovanillic acid were both present in the sample described above, only syringaldehyde was detected. As would be expected, only the most abundant analytes (or those with extremely high response factors) would typically be observed via this technique owing to the inherent qualities of DIESI. Second, although a number of analytes with identical chemical formulas have cross sections that are significantly different, to the extent that analytical assignments are possible, it is noted that the resolving power of the instrument prohibits baseline (or even partial) resolution if these analytes were to occur simultaneously. Although this is a limitation of the physical properties of the instrumentation employed in this work (which has an IMS resolving power of $~ 4-7$ ), we note that commercially available instrumentation offer mobility separations with improved resolving power $\left(\mathrm{R} \sim 45\left[\Omega / \Delta \Omega_{1 / 2}\right.\right.$; where $\Omega=$ ion drift times]) [47] that would be capable of separating many of these isomers. However, even at current resolution, analyte arrival-time peak width and shape (i.e., Gaussian versus non-Gaussian) can be relied on when assessing peak purity.

\section{Conclusions}

Owing to its ability to isolate relevant biomass signal from interfering sample components, DIESI-IM-HRMS presents as an attractive technique for assessing total molecular composition, which may ultimately provide a more thorough understanding of the correlation between hydrolysate composition and biofuel production. Although samples in biofuel process streams are typically analyzed for a handful of components generally occurring at less than $250 \mathrm{~m} / \mathrm{z}$, this work reveals highly complex samples with molecular weights of ions ranging up to $1500 \mathrm{~m} / \mathrm{z}$. With a 4-fold increase in peak capacity, IM-HRMS offers improved separation over HRMS alone. Increased sensitivity and decreased spectral complexity can be achieved via trendline-refined IM-HRMS, enabling useful data collection without requiring extensive sample clean-up steps prior to MS analysis. In addition, ion-neutral collision cross sections provide a degree of analyte confirmation that supersedes analyte chromatography retention time (i.e., cross section is a constant for a given analyte independent of instrumental conditions). Although the resolving power of the mobility separation was not sufficient to provide baseline resolution for analytes of very similar size and shape, baseline resolution was generally attainable with both IMS and HRMS separations combined. We note that current commercially available instrumentation offers an order of magnitude increase in mobility resolving power, which would substantially improve isomeric separation and identification in bioprocess samples. To our knowledge, these data represent the first report of IM-HRMS data of carbohydrates and other common biomass analytes in negative mode. 


\section{Acknowledgments}

The authors gratefully acknowledge the Baylor University Mass Spectrometry Center for support provided during the course of this work. This project was supported by the U.S. Department of Energy, Energy Efficiency, and Renewable Energy Division (grant number GO88074).

\section{References}

1. Waldron, K.W. (ed.): Bioalcohol Production: Biochemical Conversion of Lignocellulosic Biomass, p. 496. CRC Press, Boca Raton (2010)

2. Kelley, S.S., Rials, T.G., Snell, R., Groom, L.H., Sluiter, A.: Use of Near Infrared Spectroscopy to Measure the Chemical and Mechanical Properties of Solid Wood. Wood Sci. Technol 38, 257-276 (2004)

3. Nkansah, K., Dawson-Andoh, B.: Rapid Characterization of Biomass using Fluorescence Spectroscopy Coupled with Multivariate Data Analysis. I. Yellow Poplar (Liriodendron tulipifera L.). J Renew Sustain Energ 2, 023103 (2010)

4. Kobayashi, T., Kohn, B., Holmes, L., Faulkner, R., Davis, M., Maciel, G.E.: Molecular-Level Consequences of Biomass Pretreatment by DiluteSulfuric Acid at Various Temperatures. Energy Fuel 25, 1790-1797 (2011)

5. Helm, R.F., Jervis, J., Ray, W.K., Willoughby, N., Irvin, B., Hastie, J., Schell, D.J., Nagle, N.: Mass Spectral Analyses of Corn Stover Prehydrolysates to Assess Conditioning Processes. J. Agric. Food Chem 58, 12642-12649 (2010)

6. Chen, S.-F., Mowery, R.A., Castleberry, V.A., van Walsum, G.P., Chambliss, C.K.: High-Performance Liquid Chromatography Method for Simultaneous Determination of Aliphatic Acid, Aromatic Acid and Neutral Degradation Products in Biomass Pretreatment Hydrolysates. $J$. Chromatogr. A 1104, 54-61 (2006)

7. Humpula, J.F., Chundawat, S.P.S., Vismeh, R., Jones, A.D., Balan, V., Dale, B.E.: Rapid Quantification of Major Reaction Products Formed during Thermochemical Pretreatment of Lignocellulosic Biomass using GC-MS. J. Chromatogr. B 879, 1018-1022 (2011)

8. Fenske, J.J., Griffin, D.A., Penner, M.H.: Comparison of Aromatic Monomers in Lignocellulosic Biomass Prehydrolysates. J. Ind. Microbiol Biotechnol 20, 364-368 (1998)

9. Sharma, L.N., Becker, C., Chambliss, C.K.: Analytical Characterization of Fermentation Inhibitors in Biomass Pretreatment Samples using Liquid Chromatography, UV-Visible Spectroscopy, and Tandem Mass Spectrometry. In: Mielenz, J.R. (ed.) Biofuels: Methods and Protocols, p. 125. Humana Press, Totowa, NJ (2009)

10. Luo, C., Brink, D.L., Blanch, H.W.: Identification of Potential Fermentation Inhibitors in Conversion of Hybrid Poplar Hydrolysate to Ethanol. Biomass Bioenergy 22, 125-138 (2002)

11. Sevcik, R.S., Mowery, R.A., Becker, C., Chambliss, C.K.: Rapid Analysis of Carbohydrates in Aqueous Extracts and Hydrolysates of Biomass using a Carbonate-Modified Anion-Exchange Column. J. Chromatogr. A 1218, 1236-1243 (2011)

12. Chundawat, S.P.S., Vismeh, R., Sharma, L.N., Humpula, J.F., da Costa Sousa, L., Chambliss, C.K., Jones, A.D., Balan, V., Dale, B.E. Multifaceted Characterization of Cell Wall Decomposition Products Formed during Ammonia Fiber Expansion (AFEX) and Dilute-AcidBased Pretreatments. Bioresource Technol 101, 8429-8438 (2010)

13. Guignard, C., Jouve, L., Bogéat-Triboulot, M.B., Dreyer, E., Hausman, J., Hoffman, L.: Analysis of Carbohydrates in Plants by High-Performance Anion-Exchange Chromatography Coupled with Electrospray Mass Spectrometry. J. Chromatogr. A 1085, 137-142 (2005)

14. Becker, C., Sharma, L.N., Chambliss, C.K.: Analytical Monitoring of Pretreatment and Hydrolysis Processes in Lignocellulose-to-Bioalcohol Production. In: Waldron, K.W. (ed.) Bioalcohol Production: Biochemical Conversion of Lignocellulosic Biomass, p. 281. CRC Press, Boca Raton (2010)

15. Liu, X., Plasencia, M., Ragg, S., Valentine, S.J., Clemmer, D.E. Development of High Throughput Dispersive LC-Ion Mobility-TOFMS Techniques for Analyzing the Human Plasma Proteome. Brief. Funct. Genomic. Proteomic 3, 177-186 (2004)

16. McLean, J.A., Ruotolo, B.T., Gillig, K.J., Russell, D.H.: Ion Mobility-Mass Spectrometry: A New Paradigm for Proteomics. Int. J. Mass Spectrom 240, 301-315 (2005)
17. Valentine, S.J., Plasencia, M.D., Liu, X., Krishnan, M., Naylor, S. Udseth, H.R., Smith, R.D., Clemmer, D.E.: Toward Plasma Proteome Profiling with Ion Mobility-Mass Spectrometry. J. Proteome Res 5, 2977-2984 (2006)

18. Ahmed, A., Cho, Y.J., No, M., Koh, J., Tomczyk, N., Giles, K., Yoo, J.S., Kim, S.: Application of the Mason-Schamp Equation and Ion Mobility Mass Spectrometry to Identify Structurally Related Compounds in Crude Oil. Anal. Chem 83, 77-83 (2011)

19. Becker, C., Qian, K., Russell, D.H.: Molecular Weight Distributions of Asphaltenes and Deasphaltened Oils Studied by Laser Desorption Ionization and Ion Mobility Mass Spectrometry. Anal. Chem 80, 85928597 (2008)

20. Becker, C., Fernandez-Lima, F.A., Russell, D.H.: Ion Mobility-Mass Spectrometry: A Tool for Characterizing the Petroleome. Spectroscopy 24, 38-42 (2009)

21. Dwivedi, P., Schultz, A.J., Hill Jr., H.H.: Metabolic Profiling of Human Blood by High-Resolution Ion Mobility Mass Spectrometry (IM-MS). Int. J. Mass Spectrom 298, 78-90 (2010)

22. Chan, E.C.Y., New, L.S., Yap, C.W., Goh, L.T.: Pharmaceutical Metabolite Profiling using Quadrupole/Ion Mobility Spectrometry/ Time-of-Flight Mass Spectrometry. Rapid Commun. Mass Spectrom 23, 384-394 (2009)

23. Bedair, M., Sumner, L.W.: Current and Emerging Mass-Spectrometry Technologies for Metabolomics. Trends Anal. Chem 27, 238-250 (2008)

24. Knapman, T.W., Berryman, J.T., Campuzano, I., Harris, S.A., Ashcroft, A.E.: Considerations in Experimental and Theoretical Collision CrossSection Measurements of Small Molecules using Traveling Wave Ion Mobility Spectrometry-Mass Spectrometry. Int. J. Mass Spectrom 298, 17-23 (2010)

25. Dwivedi, P., Bendiak, B., Clowers, B.H., Hill Jr., H.H.: Rapid Resolution of Carbohydrate Isomers by Electrospray Ionization Ambient Pressure Ion Mobility Spectrometry-Time-of-Flight Mass Spectrometry (ESI-APIMS-TOFMS). J. Am. Soc. Mass Spectrom 18, 1163-1175 (2007)

26. Clowers, B.H., Dwivedi, P., Steiner, W.E., Hill Jr., H.H., Bendiak, B.: Separation of Sodiated Isobaric Disaccharides and Trisaccharides using Electrospray Ionization-Atmospheric Pressure Ion MobilityTime of Flight Mass Spectrometry. J. Am. Soc. Mass Spectrom 16, 660-669 (2005)

27. Fenn, L.S., McLean, J.A.: Structural Resolution of Carbohydrate Positional and Structural Isomers Based on Gas-Phase Ion Mobility-Mass Spectrometry. J. Phys. Chem., Chem. Phys 13, 2196-2205 (2011)

28. Fernandez-Lima, F.A., Becker, C., McKenna, A.M., Rodgers, R.P., Marshall, A.G., Russell, D.H.: Petroleum Crude Oil Characterization by IMS-MS and FTICR MS. Anal. Chem 81, 9941-9947 (2009)

29. Weston, D.J., Bateman, R., Wilson, I.D., Wood, T.R., Creaser, C.S.: Direct Analysis of Pharmaceutical Drug Formulations using Ion Mobility Spectrometry/Quadrupole-Time-of-Flight Mass Spectrometry Combined with Desorption Electrospray Ionization. Anal. Chem 77, 7572-7580 (2005)

30. Eiceman, G.A., Blyth, D.A., Shoff, D.B., Snyder, A.P.: Screening of Solid Commercial Pharmaceuticals using Ion Mobility Spectrometry. Anal. Chem 62, 1374-1379 (1990)

31. Dwivedi, P., Wu, C., Matz, L.M., Clowers, B.H., Siems, W.F., Hill Jr., H.H.: Gas-Phase Chiral Separations by Ion Mobility Spectrometry. Anal. Chem 78, 8200-8206 (2006)

32. McCooeye, M., Ding, L., Gardner, G.J., Fraser, C.A., Lam, J., Sturgeon, R.E., Mester, Z.: Separation and Quantitation of the Stereoisomers of Ephedra Alkaloids in Natural Health Products using Flow InjectionElectrospray Ionization-High Field Asymmetric Waveform Ion Mobility Spectrometry-Mass Spectrometry. Anal. Chem 75, 2538-2542 (2003)

33. Dwivedi, P., Hill Jr., H.H.: A Rapid Analytical Method for Hair Analysis using Ambient Pressure Ion Mobility Mass Spectrometry with Electrospray Ionization (ESI-IMMS). Int. J. Ion Mobil. Spectom 11, 61-69 (2008)

34. Steiner, W.E., Clowers, B.H., Matz, L.M., Siems, W.F., Hill Jr., H.H.: Rapid Screening of Aqueous Chemical Warfare Agent Degradation Products: Ambient Pressure Ion Mobility Mass Spectrometry. Anal. Chem 74, 4343-4352 (2002)

35. Ewing, R.G., Atkinson, D.A., Eiceman, G.A., Ewing, G.J.: A Critical Review of Ion Mobility Spectrometry for the Detection of Explosives and Explosive Related Compounds. Talanta 54, 515-529 (2001)

36. Asbury, G.R., Wu, C., Siems, W.F., Hill Jr., H.H.: Separation and Identification of Some Chemical Warfare Degradation Products using Electrospray High Resolution Ion Mobility Spectrometry with Mass Selected Detection. Anal. Chim. Acta 404, 273-283 (2000) 
37. Lee, D.S., Wu, C., Hill Jr., H.H.: Detection of Carbohydrates by Electrospray Ionization-Ion Mobility Spectrometry Following Microbore High-Performance Liquid Chromatography. J. Chromatogr. A 822, 1-9 (1998)

38. Zhu, M., Bendiak, B., Clowers, B., Hill Jr., H.H.: Ion Mobility-Mass Spectrometry Analysis of Isomeric Carbohydrate Precursor Ions. Anal. Bioanal. Chem 394, 1853-1867 (2009)

39. Liu, Y., Clemmer, D.E.: Characterizing Oligosaccharides using Injected-Ion Mobility/Mass Spectrometry. Anal. Chem 69, 2504-2509 (1997)

40. Um, B.; Aghazadeh, M.; van Walsum, G.P.: A Microassay to Evaluate Inhibition of Enzymatic Hydrolysis Following Dilute-Acid Pretreatment of Corn Stover. Presented at the 32nd Symposium on Biotechnology for Fuels and Chemicals, Clearwater, FL, April 2010; Poster 3-44.

41. Wolff, J.-C., Eckers, C., Sage, A.B., Giles, K., Bateman, R.: Accurate Mass Liquid Chromatography/Mass Spectrometry on Quadrupole Orthogonal Acceleration Time-of-Flight Mass Analyzers using Switching between Separate Sample and Reference Sprays. 2. Applications using the Dual-Electrospray Ion Source. Anal. Chem 73, 2605-2612 (2001)
42. de Laeter, J.R., Böhlke, J.K., de Bièvre, P., Hidaka, H., Peiser, H.S., Rosman, K.J.R., Taylor, P.D.P.: Atomic Weights of the Elements: Review 2000. Pure Appl. Chem 75, 683-800 (2003)

43. Ruotolo, B.T., Benesch, J.L.P., Sandercock, A.M., Hyung, S.J., Robinson, C.V.: Ion Mobility-Mass Spectrometry Analysis of Large Protein Complexes. Nat. Protoc 3, 1139-1152 (2008)

44. Chen, S.-F., Mowery, R.A., Sevcik, R.S., Scarlata, C.J., Chambliss, C.K.: Compositional Analysis Water-Soluble Materials in Switchgrass. J. Agric. Food Chem 58, 3251-3258 (2010)

45. Klinke, H.B., Ahring, B.K., Schmidt, A.S., Thomsen, A.B.: Characterization of Degradation Products from Alkaline Wet Oxidation of Wheat Straw. Bioresource Technol 82, 15-26 (2002)

46. Sluiter, J.B., Ruiz, R.O., Scarlata, C.J., Sluiter, A.D., Templeton, D.W.: Compositional Analysis of Lignocellulosic Feedstocks. 1. Review and Description of Methods. J. Agric. Food Chem 58, 9043-9053 (2010)

47. Giles, K., Williams, J.P., Campuzano, L.: Enhancements in Traveling Wave Ion Mobility Resolution. Rapid Comm Mass Spectrom, 15591566 (2011)

48. Wyttenbach, T., von Helden, G., Bowers, M.T.: Gas-Phase Confirmation of Biological Molecules: Bradykinin. J. Am. Chem. Soc 118, 8355-8364 (1996) 